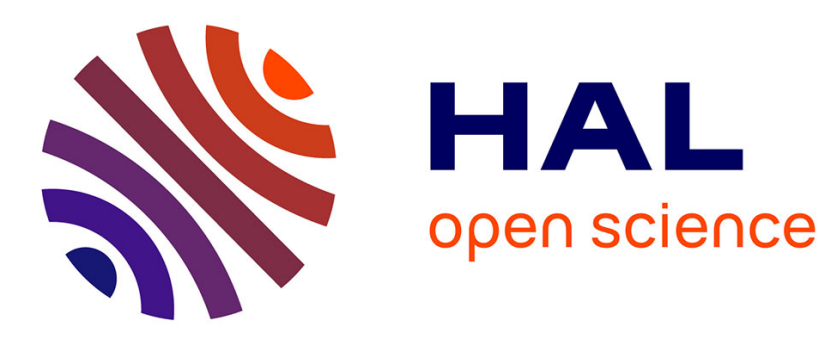

\title{
An Ultra-Wide Band Umbilical Cord for Cognitive Radio Systems
}

Christophe Moy, Alexis Bisiaux, Stéphane Paquelet

\section{To cite this version:}

Christophe Moy, Alexis Bisiaux, Stéphane Paquelet. An Ultra-Wide Band Umbilical Cord for Cognitive Radio Systems. IEEE PIMRC'05, 2005, Berlin, Germany. hal-00084151

\section{HAL Id: hal-00084151 \\ https://hal.science/hal-00084151}

Submitted on 5 Jul 2006

HAL is a multi-disciplinary open access archive for the deposit and dissemination of scientific research documents, whether they are published or not. The documents may come from teaching and research institutions in France or abroad, or from public or private research centers.
L'archive ouverte pluridisciplinaire HAL, est destinée au dépôt et à la diffusion de documents scientifiques de niveau recherche, publiés ou non, émanant des établissements d'enseignement et de recherche français ou étrangers, des laboratoires publics ou privés. 


\title{
An Ultra-Wide Band Umbilical Cord for Cognitive Radio Systems
}

\author{
Christophe Moy, Alexis Bisiaux, Stéphane Paquelet
}

\begin{abstract}
This paper describes how Cognitive Radio (CR) could benefit from Software-Defined Radio (SDR) compatible Ultra-Wide Band (UWB) systems. It introduces the notion of umbilical cord that can keep a CR device connected to its surrounding world, thanks to sensing and low speed over-the-air reconfiguration (OTAR) means provided by Low-Data Rate (LDR) systems, and to fast OTAR downloading facilities based on High-Data Rate (HDR) hot spots. A particular UWB architecture supporting SDR-compatible technological constraints is proposed as candidate to realize this promising combination of capabilities.
\end{abstract}

Index Terms - cognitive radio, ultra-wide band, softwaredefined radio, impulse radio

\section{INTRODUCTION}

$\mathrm{S}$ INCE the introduction of the wireless telephony, the terminal configuration has been under the responsibility of the user (world-wide roaming with GSM still requires the user to manually switch frequency bands when traveling from one continent to another). Next step will pass a new stage: the one of intelligent terminal. That is to say, a terminal will be capable of taking initiatives at all levels of its functionality transparently to the user, at the application level as well as at the radio level. This is known as the Cognitive Radio (CR) concept.

The ability of the lower layers of a wireless system to support multiple communication schemes includes a very wide spectrum of topics already in the scope of the SoftwareDefined Radio (SDR) research community, among them we can just evoke: concepts [1], regulatory [2], HW and SW architecture [3], reconfiguration [4], design methodology [5], signal processing [6], HW components... All those approaches, gathered under the term SDR, tend to converge towards a common goal: facilitate the design, production and commercial success of multi-purpose communication systems as they are considered as keys to answer future wireless systems paradigm.

Manuscript received March 20, 2005.

C. Moy is with Supelec, SCEE Laboratory, Cesson-Sévigné, France (phone: +33 $2099 \quad 84 \quad 45 \quad 34$; fax: +33 $2299 \quad 84 \quad 45 \quad 99$; e-mail: Christophe.Moy@rennes.supelec.fr), a lab of the IETR, UMR 6164 of CNRS.

A. Bisiaux \& S. Paquelet are with Mitsubishi Electric ITE, Rennes, France. (e-mail: bisiaux@tcl.ite.mee.com, paquelet@tcl.ite.mee.com).
This paper introduces the notion of an umbilical cord that can keep a CR device constantly connected to its environment, by resorting to the specific characteristics of Ultra-Wide Band (UWB) systems regarding sensing and downloading means. Furthermore, gathering various types of UWB technologies and applications and combining them with CR bring a new light onto the promising field of ubiquitous radio systems.

The paper is organized as follows: Cognitive Radio, its common features, requirements and challenges, are presented in part II. Part III describes how the UWB technology could be useful in a CR context. Finally, the umbilical cord concept is detailed in part IV, along with a particular UWB architecture that could be used in that frame.

\section{Cognitive Radio}

\section{A. Overview}

The Cognitive Radio concept, here tackled in its widest and unrestricted sense, refers to a communication system that is able to observe its environment, analyze it, and react to it in some way. This kind of control loop is illustrated in Fig. 1.

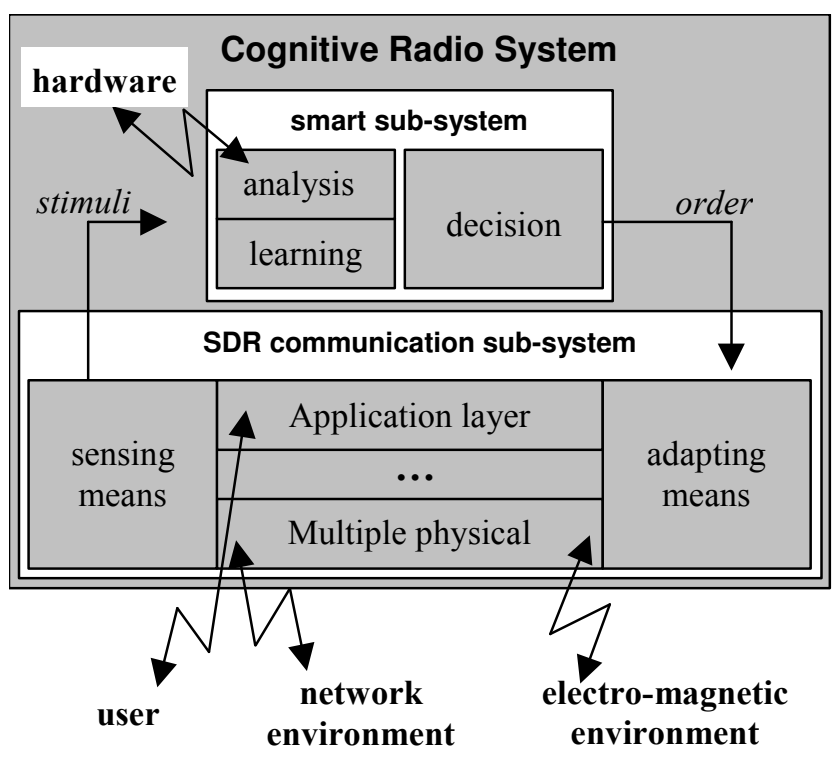

Fig.1 schematic view of a Cognitive Radio system

In addition to its multiple communication parts, the CR system features sensing means, adapting means, and a smart sub-system dedicated to analyzing stimuli and taking decisions. By properly combining them, the system can 
acquire, to some extent, both the self-awareness and autonomy it needs to automatically take initiatives that will make life easier (or cheaper) for its owner, without even his being aware of it. How much cognitive the device is in the end depends on those three components. In order to be consistent, the analysis and learning parts of the smart sub-system must be fed with sufficient stimuli, and its decision part must make sure that the underlying hardware can indeed support its adaptation orders.

\section{B. Sensing means}

Sensing means refer to all the possible methods the CR system has at its disposal for observing its environment, which can be categorized in four main families represented in Fig.1 with lightning arrows:

- electromagnetic environment: spectrum occupancy, Signal to Noise Ratio (SNR), multi-paths propagation...

- hardware environment: battery level, power consumption...

- network environment: telecommunication standards (GSM, UMTS, WiFi, etc.), operators and services available in the vicinity, traffic load on a link...

- user-related environment: position, speed, time of day; user preferences, user profile (access rights, contract...), video and audio sensor (presence detection, voice recognition)...

As shown in Fig.1, all the stimuli received by the sensing means of the different layers (from physical to application) must be merged and analyzed together to yield the best possible decision of the smart sub-system. The range of possibilities, as well as the high level of complexity involved, can be grasped through a simple example. Somebody is walking down the street, watching a football game on his CR terminal; the terminal detects that the battery is quickly emptying itself, probably because it is too far from the base station (low SNR, high transmit power). By comparing its current geographical position with information gathered from the network about the surrounding telecommunication infrastructure, it is able to tell the user where to go to obtain a better transmission that will save battery life and maybe allow him to watch the end of the game.

Whereas some of the sensing means mentioned above already exist (the less sophisticated ones like battery level or SNR measurement), some others may have to wait a bit before becoming a reality. One of the hottest research topics in the Cognitive Radio field today is spectrum management. With the arrival of new wireless telecommunication standards on the one hand, and the ever increasing number of users on the other, the spectrum is gradually becoming saturated. A current trend is to find a way of better using frequency resources through time and space [7]. The solution partly lies in systems that will be capable of dealing with a plurality of bands, if not a completely flexible RF front-end. A regulatory policy that would no more restrain standards to pre-determined operation bands, or would generalize unlicensed bands is a prerequisite.

Another very promising feature of Cognitive Radio would be its ability to determine its geographical position. In addition to the usual services that an average GPS set can offer [8], such a positioning ability, coupled with a telecommunication network, would bring a wide range of new services to the user and as many value-added activities to operators. It could first of all reinforce GPS's coverage and precision and benefit from centralized maps databases to virtually explore its geographical environment. Also, by locating other CR systems in the vicinity, be they mobile or not, either by direct radio contact or by searching the network, it could gather various kinds of evanescent information that no remotely stored database could provide: detecting the presence of a friend, a taxi in the next street, or a high data-rate hot spot installed just around the corner for a limited time.

\section{Adapting means}

The football game example given in the previous section illustrates how the sophisticated sensing means of a CR terminal can be of valuable help to its user, yet it remained his responsibility to react in the most appropriate way (take a few steps toward a base station in that case). The possibilities of such an intelligent communication system should extend far beyond that however, and provide automatic procedures to adapt to its environment in an efficient way, transparently to its user.

The actions the CR system can take in that purpose can in turn be categorized in several classes depending on the number of actors involved in the process (terminal, network, operator, regulation, manufacturer, user). Furthermore, several subclasses can be identified in each case with different complexity implications (static, dynamic, cooperation with other actors):

- terminal level: bug fixing, upgrade of low-level code (static); modification of a signal processing algorithm depending on propagation conditions or on battery level (dynamic)...

- system level (terminal, network, operator, regulation): frequency switching (static or dynamic), handover from one communication standard to another (dynamic)...

- application level (terminal, operator, user): bug fixing, upgrade of application-level code (static), download ad-hoc applications (dynamic)...

All those adapting actions involve reconfiguration mechanisms whose complexity depends on the amount of objects to reconfigure, the speed at which this reconfiguration must operate, and the actors involved in the process. In order to have a constant access to a remote network in charge of managing reconfiguration, a ubiquitous dedicated channel may be necessary to download configuration data "over the air", either while on-line or off-line, for static or dynamic reconfiguration, and at physical layer up to application layer.

As an example, a terminal running UMTS detects that it is getting closer to a base station where SNR is better and decides to use sub-optimal signal processing algorithms that will still guarantee the required quality of service while consuming less power. Those new algorithms can be dynamically downloaded as needed [9]. As it goes by, the terminal detects that it is entering a WiFi-covered area and decides to switch to that standard in order to benefit from cheaper billing rates, according to its owner's preferences. Finally, the terminal takes this high-data-rate WiFi opportunity 
to get a better-quality video stream with larger image size and greater definition, after having downloaded an appropriate video player if needed.

\section{UWB FOR COGNITIVE RADIO SYSTEMS}

\section{A. UWB perspectives}

CR systems must combine both multiple sensing means and a flexible architecture, which pleads in favor of an SDR design approach. Moreover, multi-standard capability is required for both communication (original function) and sensing purposes to retrieve information from the environment through radio means. This section explains how providing UWB facilities to a CR device could answer those issues.

Even though UWB communication systems are not yet operational, it can be predicted, judging by the craze that this technology has been arousing for several years now, that they will soon become an inevitable part of our every day life. It is difficult to give a single definition of UWB however, because it is destined to span a very wide range of applications field that extends even beyond the traditional borders of telecommunications themselves. UWB is often mentioned with accompanying terms such as radar, localization, sounding, medical instrumentation, sensor networks, RFID, cable replacement... Yet two main trends can be identified: LowData-Rates (LDR) UWB systems (hundreds of Kbps) target low-cost, low-consumption applications with ranging capabilities. High-Data-Rates (HDR) and Very High Data Rates (VHDR) UWB systems (from several Mbps up to $1.6 \mathrm{Gbps})$ are meant to help throughput-intensive devices go wireless.

UWB is traditionally defined as an impulse-based transmission method. The energy of such short impulses spreads over a very wide band, so that its power spectral density is very low. Discreet as it is, the UWB signal can spread over frequency bands already reserved for well established communication standards without jamming them. In 2002, the FCC issued a recommendation allowing licensefree UWB systems to operate between 3.1 and $10.6 \mathrm{GHz}$ provided that they did not exceed $-41.25 \mathrm{dBm} / \mathrm{MHz}$.

Besides, engineers who tackle UWB soon come to face some very tricky technological difficulties; VHDR solutions that target more than $1 \mathrm{Gbps}$ must have the processing power to support that; LDR solutions need to track in a very precise way pulses shorter than a nanosecond in order to perform efficient localization. In all cases, low transmitted power and possible in-band interferors (narrow-band systems) make it hard to detect and track the signal.

As can be seen from those considerations, UWB, just like Cognitive Radio, is not yet totally operational, but good hopes can be had for the future. The next two sections describe how UWB, without focusing on any particular technology, could meet many of the requirements concerning Cognitive Radio as listed in part II.

\section{B. UWB sensing means}

It is foreseen that at least some categories of UWB devices will have to be able to scan the whole 3.1 to $10.6 \mathrm{GHz}$ spectrum in search of free sub-bands to transmit into. This is particularly true for VHDR devices that cannot afford to transmit data over frequencies already being used by narrowband systems. Not only might they be a nuisance to them, but they will also very probably be completely jammed by them. LDR systems (and possibly HDR) can avoid that problem by using frequency and/or time diversity methods, but some of them might still be able to analyze the spectrum. When available, this scanning capacity can prove very useful in a Cognitive Radio context, to help the CR system explore its electromagnetic environment and even make a guess at what other telecommunication standards are up in the vicinity.

Another expected powerful feature of UWB is its ranging ability. A UWB system, on its own, can potentially work as a radar or sounder by emitting pulses and listening to their echoes. But UWB is at first meant for telecommunications, and it would just make sense that it would use this primary capability to determine its precise location. With a sufficiently dense population of UWB devices (the "sufficient density" depending on their range, possibly several tens of meters), any mobile UWB system could know its absolute position continuously. Not only that, but it would also be able to evaluate its speed and trajectory, and the ones of its neighbors. Unlike GPS-based services that provide static geographical information stored in databases, UWB can gather data on the fly and build up a dynamic map of its environment.

A vast application field targeted by UWB is the one of sensor networking. With its communicating and ranging capabilities, its low cost and low power consumption, UWB is expected to extend far beyond the current limits of sensor networks and tackle new applications, most of which have not even been imagined yet: remote identification modules, staff identification badges, anti-collision radar, remote temperatureor light-sensitive sensors... Those applications will very probably become the major contributors to the spreading of UWB systems. A CR device could greatly benefit from the information directly provided by such remote sensors without their having to be integrated in the device itself.

\section{Ubiquitous downloading channel}

As mentioned in section II.C, sophisticated CR systems with reconfiguration capability must maintain a constant connection to remote databases in order to download pieces of code as needed. Supposing those systems supported UWB to benefit from its powerful sensing means, they could as well use its communication means to download configuration data. Resorting to an already stated hypothesis, with a sufficiently dense population of UWB devices a CR system could obtain a ubiquitous connection to such configuration-specific links. Configuration information would then always pass through the same UWB channel.

The throughput that can be achieved over that channel largely depends on the UWB system's characteristics. It is 
predictable that ubiquitous connection will be obtained through LDR devices rather than HDR most of the time. First of all because their low cost and low power consumption should favor their deployment; but also because they are supposed to cover a larger area, at the price of a lower throughput. LDR systems are expected to achieve transmission rates of about $100 \mathrm{kbps}$ at $100 \mathrm{~m}$, against $100 \mathrm{Mbps}$ at $10 \mathrm{~m}$ for HDR systems. Hence CR devices would get different reconfiguration services depending on the nature of the surrounding UWB systems. LDR would be there most of the time to provide basic ones for which downloading time is not critical, like bug fixing, code enhancement, or even downloading of a new air interface yet unknown to the CR device (it might be worth waiting a few seconds to download it if it is the only available high data-rate standard in the vicinity). Here and there, HDR UWB systems would provide local hot spots where more demanding reconfiguration scenarios would be workable, like dynamic code adaptation or handover from one communication standard to another.

These statements rely on the assumption that the CR device can accommodate to most UWB standards it might encounter, of both LDR and HDR types. In other words, it implies that the UWB system itself is flexible, either by nature (multipurpose modulation scheme), or through reconfiguration capabilities, or both. Without going that far, next section describes a particular UWB air interface that supports the SDR features required to obtain such a flexibility and provide an umbilical cord to the CR system.

\section{A UWB UMBILICAL CORD}

\section{A. Definition}

The umbilical cord should be here understood as the link that keeps a CR device permanently connected to its surrounding world, that feeds it will all the nutrients it needs to evolve, and that provides the information its cognitive nature demands. The various points developed in section III show that UWB could perfectly play that role. This idea is illustrated in Fig.2, where UWB is used for both its highly versatile sensing means (spectrum occupancy, positioning) and its communication means (data downloading for remote reconfiguration).

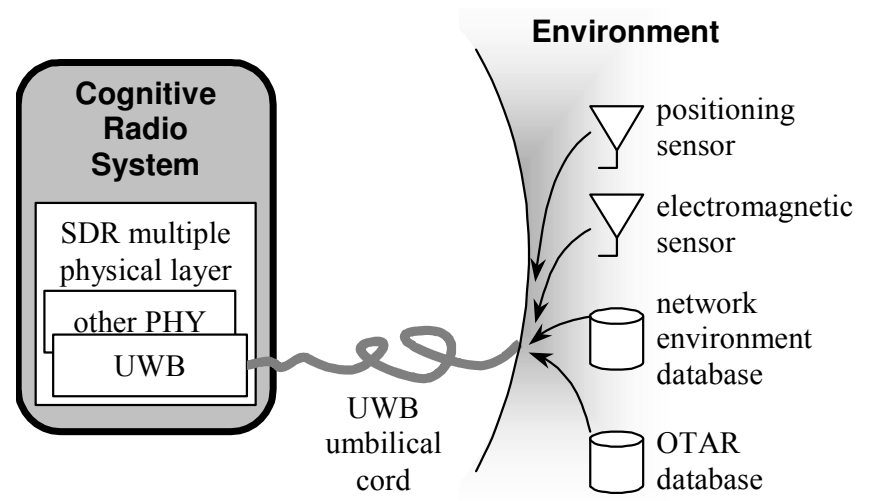

Fig.2 UWB umbilical cord for CR systems
Of course, the thickness of the umbilical cord depends on the types of UWB systems that are available in the vicinity: LDR systems will mostly provide sensing information at low speed, whereas HDR systems will be more useful when it comes to downloading configuration data at high speed. A combination of both types would bring the CR device the best ubiquitous connectivity to its environment.

In order to maintain a constant connection to the surrounding world, the umbilical cord must be able to adapt itself to the various kinds of UWB systems it encounters. In that purpose, de CR device's UWB air interface must itself possess intrinsic flexibility properties and support SDR's basic reconfigurability principles. This can prove hard to achieve though. Indeed, SDR guidelines recommend to sample the signal as close to the antenna as possible and then let digital chips do the rest of the processing for flexibility and adaptability's sake; but digitizing, and then processing, a signal that spans more than $2 \mathrm{GHz}$ of band is very demanding in terms of sampling speed, computing resources and power consumption, and implementing it in a mobile handset can simply not be envisaged as of today's state-of-the-art technologies. Therefore, not all UWB solutions are good candidates for being implemented in an SDR fashion.

\section{B. A compatible UWB solution}

This section presents an HDR/VHDR UWB architecture designed in the spirit of SDR, although with quite an unusual approach that makes its implementation feasible at low cost and compatible with SDR technological constraints. It shows how the HDR structure is in adequacy with the umbilical cord paradigm.

The HDR structure is based on a non-coherent energy detection method previously described in more details in [10]. Data are transmitted over several $250-\mathrm{MHz}$-wide sub-bands (up to 24 to cover the whole spectrum) with a simple pulsebased On/Off Keying (OOK) modulation. Pulses are transmitted at time intervals slightly longer than the channel's delay spread to avoid inter-symbol interference.

The demodulation process consists in measuring the energy received in each sub-band and comparing it to a previously estimated threshold as explained in Fig.3. Due to the very short duration of pulses, there is no significant intra-symbol interference due to multi-path propagation. Rather, all received pulses add up to contribute to the overall measured energy.

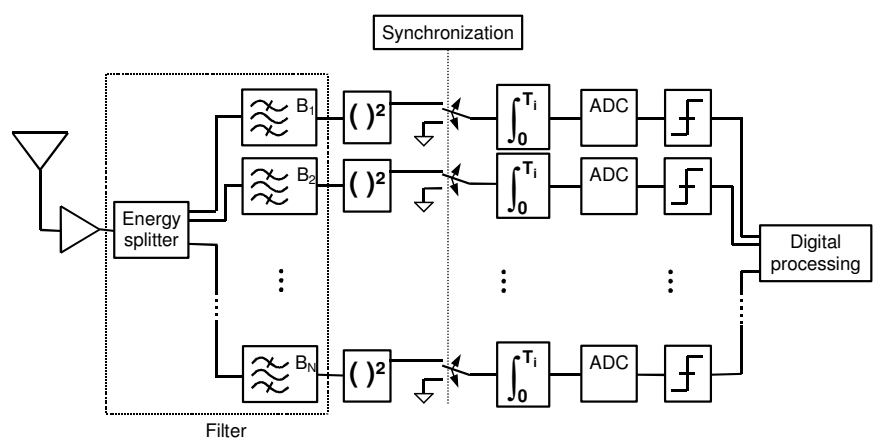

Fig.3 RF front-end of the VHDR UWB receiver 
Performance results [10] based on IEEE channel models show that data rates of up to $600 \mathrm{Mbps}$ could be achieved at $3 \mathrm{~m}$ and with an error probability of $10^{-5}$ (without coding). Even higher throughputs are expected in good propagation conditions (delay spread shorter than $40 \mathrm{~ns}$ ) or at shorter range.

\section{SDR \& CR features}

Contrary to regular SDR implementation, the receiver resorts to an advanced analog front-end that pre-processes the UWB signal in such a way that its fundamental properties (the information it carries) remain virtually unaffected. Hence the channel need not be sampled at the Nyquist rate (several $\mathrm{GHz}$ ): it is sufficient to take a single sample per transmitted pulse, in order to extract all the necessary energetic information. With typical delay spreads of $10 \mathrm{~ns}$ up to $100 \mathrm{~ns}$, pulse repetition rates range from $10 \mathrm{MHz}$ to $100 \mathrm{MHz}$, making the receive sampling rate not exceed 100 MSPS. The resulting signal can then be processed by the digital back-end at a much lower rate than other HDR UWB solutions like MB-OFDM and DS-UWB. This allows the system to support SDR features that could not be envisaged otherwise and gives the digital back-end a chance to adapt itself to the multiple purposes of the umbilical cord.

To compensate for the relatively low rate at which pulses are emitted (several tens of $\mathrm{MHz}$, as imposed by the channel delay spread), the signal is split into 24 sub-bands that all carry 1 bit of data. Not only does this parallel structure permit to achieve very high throughputs, but it also possesses intrinsic Cognitive Radio properties. Indeed, by measuring the energy received in each sub-band, the UWB device is able to dynamically analyze the spectrum's occupancy in steps of $250 \mathrm{MHz}$, and possibly detect the presence of nearby narrowband systems. It can then easily switch off any polluted band as needed to maintain a good link budget at the cost of a minor loss of throughput, while keeping from interfering with other systems. This built-in frequency scanning faculty could as well be used by the CR device to analyze its electromagnetic environment at any time, thus adding a sensing capability to this fast-download umbilical cord.

Finally, the VHDR UWB system's architecture, that features no such critical blocks as oscillators and mixers, whose noncoherent energy detection method does not require great hardware precision nor phase linearity [11], and that relaxes constraints put on the analog to digital interface a lot, makes a good candidate for the design of cheap UWB solutions. The UWB umbilical cord based on this solution would share a unique SDR digital back-end with other communication standards, and the resulting system could target a large scale of applications and markets.

It can be anticipated from this HDR architecture that an impulse-based equivalent approach to LDR is viable, lying on a similar principle of an analog RF front-end providing the SDR digital back-end with signals sampled at rates of the same order of magnitude, as evoked in [12]. The combination of
LDR and HDR would complete the umbilical cord paradigm since both would share SDR technological compliance for adaptation needs and an impulse-based scheme. The umbilical cord would then be based on two modes:

- HDR: only available at hot spots with a short-range UWB access point, for voluminous data or immediate real-time downloading needs as well as spectrum analysis,

- LDR: ubiquitous connection to downloading means, localization ability and access to surrounding UWB sensors.

\section{CONCLUSION}

Cognitive Radio and UWB are certainly two of the hottest topics in the R\&D telecommunication community today. Their respective potential applications are countless, which explains the wide interest they have been arousing. It also explains why it will take some more time for them to become really operational: issues are numerous, at both technical and political levels, and solutions are complex. Still, it has been shown in this paper that CR and UWB are two complementary technologies that would greatly benefit from each other. The example provided in the last section shows that they are not necessarily exclusive as is often believed, and that adequate design techniques can make them coexist advantageously. On the one hand, UWB brings sensing capabilities to CR systems that would be hard to obtain with other means. On the other hand, SDR provides UWB with the flexibility it needs to support various configurations of use.

\section{REFERENCES}

[1] Joe Mitola III, The Software Radio Architecture, IEEE Communicatons Magazine, vol. 33, n5, pp 26-37, May 1995

[2] SDRF - Software Defined Radio Forum, http://www.sdrforum.org/.

[3] A. Kountouris, C. Moy, Reconfiguration in Software Radio Systems, 2nd Karlsruhe Workshop on Software Radios, Mar. 2002.

[4] A. Kountouris, C. Moy, L. Rambaud, Re-configurability: a Key Property in Software Radio Systems, 1st Karlsruhe Workshop on Software Radios, Mar. 2000.

[5] C. Moy, M. Raulet, S. Rouxel, J.P. Diguet, G. Gogniat, P. Desfray, N. Bulteau, J.E. Goubard, Y. Denef, UML Profile for Waveform Signal Processing Sub-system Abstraction, SDR Forum Technical Conference 2004, Phoenix, November 2004.

[6] H. Ishii, S. Kawamura, T. Suzuki, M. Kuroda, H. Hosoya, H. Fujishima, An Adaptive Receiver based on Software Defined Radio Techniques, Proc. 12th PIMRC, USA, vol.2, pp. 120-124, September 2001

[7] FCC NPRM 03 322, NPRM- Facilitating Opportunities for Flexible, Efficient and Reliable Spectrum Use Employing Cognitive Radio Technologies, 17 December 2003

[8] C. Roland, J. Palicot, Un Terminl Multi-Standard Utlisant le GPS pour se Reconfigurer, (in French), ISVIC'2000, Rabat, Morocco

[9] C. Moy, A. Kountouris, A. Bisiaux, $H W$ and $S W$ Architectures for Over-The-Air Dynamic Reconfiguration by Software Download, (slides) SDR Workshop of the IEEE Radio and Wireless Conference, Boston, USA, Aug. 2003

[10] S. Paquelet, L-M.Aubert and B. Uguen, An Impulse Radio Asynchronous Transceiver for High Data Rates, UWBST and IWUWBS Kyoto, May 2004.

[11] S. Paquelet, C. Moy, L-M.Aubert, RF Front-End Considerations for SDR Ultra-Wideband Communications Systems, RF Design, Jul. 2004.

[12] C. Moy, S. Paquelet, A. Bisiaux, A. Kountouris, A SDR UltraWideband Communication System for Low and High Data Rates, SDR Forum Technical Conference, Phoenix, USA, Nov. 2004. 